\title{
TQFT versus RCFT: 3-d topological invariants
}

\author{
BOGUSŁAW BRODA \\ Department of Theoretical Physics, University of Eódź \\ Pomorska 149/153, PL-90-236 Eódź, Poland
}

\begin{abstract}
A straightforward relationship between the two approaches to 3-dimensional topological invariants, one of them put forward by Witten in the framework of topological quantum field theory, and the second one proposed by Kohno in terms of rational conformal field theory, is established.
\end{abstract}

\section{Introduction}

In 1989, Witten proposed a new topological invariant of 3-dimensional manifolds $\tau_{k}(\mathcal{M})$ in the framework of topological quantum field theory (TQFT) defined by the Chern-Simons action. ${ }^{1}$ His idea has been next developed and mathematically refined by Reshetikhin and Turaev. ${ }^{2}$ Though, strictly speaking, Witten explicitly used the apparatus of TQFT (or even conformal field theory) and described 3-manifolds in terms of the Dehn (or rational) surgery, whereas Reshetikhin and Turaev applied the combinatorics of representations of quantum groups and the honest (or integer) surgery, we will identify the both methods as the Reshetikhin-Turaev-Witten (or surgical) approach. Other topological invariant of 3-manifolds, proposed by Turaev and Viro, bases on a simplicial description of 3-manifolds and is related, from physical point of view, to 3-dimensional topological gravity (see, e.g. Ref. 3). Finally, Kohno has introduced another invariant of 3-manifolds $\phi_{k}(\mathcal{M})$, which relies on the formalism of rational conformal field theory (RCFT) and the Heegaard

\footnotetext{
* e-mail: bobroda@mvii.uni.lodz.pl

PACS Nos.: 11.15.Tk, 02.40.+m.
} 
decomposition $^{4,5}$ (see also references in Ref. 5).

Thus, we have at our disposal the three kinds of 3-manifold invariants, each one associated with a different topological presentation of a 3-manifold and with different physical and/or mathematical tools:

(i) surgery - quantum Chern-Simons field theory (Witten), representations of quantum groups (Reshetikhin and Turaev);

(ii) triangulation - representations of quantum groups (Turaev and Viro), topological gravity;

(iii) Heegaard decomposition-WZW model (Kohno), representations of mapping class group.

Interestingly, it appears that all the approaches are, in fact, equivalent. The wellknown equivalence of the surgical and the simplicial invariant has been established in physical context in Ref. 3, whereas the equivalence of the surgical and the Kohno invariant in the context of quantum field theory (see Theorem 4.2 in Ref. 5) is addressed in this letter.

\section{3-d invariants}

In this section, we shortly summarize the definition of the both 3-dimensional invariants, $\tau_{k}(\mathcal{M})$ and $\phi_{k}(\mathcal{M})$ respectively.

a) tqft

The surgical presentation of a 3-manifold let us associate, highly non-uniquely (i.e. modulo the Kirby moves), to each manifold $\mathcal{M}$ a link $\mathcal{L}$. Therefore, from the point of view of $\mathcal{M}$, we should identify all the links related by means of the two Kirby moves, as they yield the same 3-manifold. To construct the topological in- 
variant of $\mathcal{M}$ we can introduce a special element of the fusion algebra related to SU(2) Chern-Simons field theory

$$
\Omega=\bigoplus_{i=0}^{k-1} d_{i} W_{i},
$$

where $k$ is a fixed positive integer (the level), $k=1,2, \ldots, W_{i}$ is an element of the fusion algebra corresponding to $i+1$ dimensional irreducible representation of $\mathrm{SU}(2)$, and $d_{i}$ is a number, the quantum dimension of $W_{i}$. Thus, in the context of SU(2) ChernSimons field theory, $\Omega$ gives rise to a linear combination of Wilson lines weighted by $d_{i}$.

The "partition function"

$$
\left\langle\Omega_{\mathcal{L}}\right\rangle
$$

with $\Omega$ assigned to each component of $\mathcal{L}$, is invariant with respect to the second Kirby move, handle slides. For further convenience, we shall slightly change the normalization of $\Omega$ defining

$$
\omega \equiv\left\langle\Omega_{\mathcal{U}}\right\rangle^{-\frac{1}{2}} \Omega
$$

where $\mathcal{U}$ is a 0 -framed unknot. After a simple re-normalization (stabilization) we obtain a true topological invariant

$$
\tau_{k}(\mathcal{M})=\frac{\left\langle\omega_{\mathcal{L}}\right\rangle}{\left\langle\omega_{\mathcal{U}_{+}}\right\rangle^{b_{+}}\left\langle\omega_{\mathcal{U}_{-}}\right\rangle^{b_{-}}}
$$

where $\mathcal{U}_{+}\left(\mathcal{U}_{-}\right)$denotes an unknot with a single positive (negative) twist, and $b_{+}\left(b_{-}\right)$ is the number of positive (negative) eigenvalues of the linking matrix $\ell k(\mathcal{L})$.

b) $r c f t$

An arbitrary 3 -manifold $\mathcal{M}$ can also be presented via a Heegaard decomposition

$$
\mathcal{M}=\mathcal{V}_{g} \cup_{h}\left(-\mathcal{V}_{g}\right)
$$


where $\mathcal{V}_{g}$ is a handlebody of genus $g$, and $h$ is a homeomorphism identifying the boundaries of the handlebodies. Let denote by $\rho_{k}$ the projective representation of the mapping class group acting on colored edges of the dual graph representing a pant decomposition of $\Sigma_{g}=\partial \mathcal{V}_{g}$, found in the context of SU(2) RCFT at the level $k$ in Ref. 6 (see also Ref. 7). Now we can introduce a "vacuum expectation value"

$$
\left\langle\rho_{k}(h)\right\rangle_{0},
$$

where the symbol " \langle\rangle$_{0}$ " means the $(0,0)$-entry of the matrix $\rho_{k}(h)$. The quantity $(5)$ is independent of the Heegaard decomposition modulo $\Gamma_{k}$, a cyclic group generated by $\kappa_{k}=\exp \left(2 \pi i \frac{c}{24}\right)$, with $c=3 k /(k+2), k=1,2, \ldots$. Finally, following Ref. 4 , we have to stabilize (5) yielding

$$
\phi_{k}(\mathcal{M})=\left\langle\omega_{\mathcal{U}}\right\rangle^{g}\left\langle\rho_{k}(h)\right\rangle_{0},
$$

a true invariant of $\mathcal{M}\left(\right.$ modulo $\left.\Gamma_{k}\right)$.

\section{Equivalence}

In this section, we will show the equivalence of the (TQFT) Chern-Simons surgical invariant $\tau_{k}(\mathcal{M})$ (Eq. 4) and the (RCFT) Heegaard decomposition invariant $\phi_{k}(\mathcal{M})$ (Eq. 6). To this end we will translate $\phi_{k}$ (basically built of a product of generators of Dehn twists) onto weighted linear combinations of Wilson loops (surgery loops). As it is well-known, every orientation-preserving homeomorphism of a closed orientable 2-manifold $\Sigma_{g}$ of genus $g$ is isotopic to a product of Dehn twist homeomorphisms along the $3 g-1$ (Lickorish) canonical curves pictured in Fig. 1 . An explicit set of generators of the Dehn twists satisfying Wajnryb's relations for genus $g$, modulo $\Gamma_{k}$, is proposed in Ref. 6 . In turn, each Dehn twist along a circle $\mathcal{U}$

can be implemented at the representation level by assigning to it $\omega_{\mathcal{u}_{ \pm}}$. Heuristically, it follows from the fact, well-known in the Fenn-Rourke version of the Kirby cal- 
culus, that $\omega_{\mathcal{U}_{+}}\left(\omega_{\mathcal{U}_{-}}\right)$twists clockwise (anti-clockwise) lines going through $\mathcal{U}_{+}\left(\mathcal{U}_{-}\right)$. It has been shown in Ref. 7 explicitly that putting $\omega_{\mathcal{U}_{ \pm}}$round an edge of the dual graph representing a pant decomposition of $\Sigma_{g}$ yields the corresponding Dehn twist (modulo $\Gamma_{k}$ ). Thus, we can assign to every product of generators of Dehn twists $T=\tau_{r} \cdots \tau_{2} \tau_{1}$ a special link denoted as $\mathcal{L}^{-1}(T)$. The link consists of a finite number of circles, each of which lies on, and is concentric with, one of the annuli of Fig. 2. The arrangement of the components of $\mathcal{L}^{-1}(T)$ follows from the order of the Dehn twists $\tau_{n}, n=1,2, \ldots, r$, furthest Dehn twists (greatest indices) correspond to outermost surgery unknots $\mathcal{U}_{ \pm}$. Proceeding this way we obtain the special link $\mathcal{L}^{-1}(T)$ corresponding to $\rho_{k}(h)$. To calculate the "vacuum expectation value" of $\rho_{k}(h)$, i.e. the $(0,0)$-entry (0 means the trivial representation), one should evaluate the "partition function" of an auxiliary link $\mathcal{U} \mathcal{L}^{-1}(T)$, derived from $\mathcal{L}^{-1}(T)$ in the following manner. Since $\rho_{k}(h)$ is to act on a dual graph (Fig. 2) colored with the trivial representation (the first 0 -entry), actually the graph vanishes due to the triviality of representations. To project out the second 0-entry we should put a 0-framed surgery unknot $\mathcal{U}$, i.e. $\omega_{\mathcal{U}}$, round each one-handle of $\Sigma_{g}$ encircling lines belonging to $\mathcal{L}^{-1}(T) . \omega_{\mathcal{U}}$ has a well-known property of cancelling all lines going through $\mathcal{U}$ except those which correspond to the trivial representation. We can note that actually the required (minimal) number of $\mathcal{U}$ 's (primary ones) is exactly equal to $g$, because temporarily we can always add some auxiliary unknot $\mathcal{U}$, and use the second Kirby move to shift it on $\Sigma_{g}$. More precisely, handle-sliding $\mathcal{U}$ along those (non-homotopic, meridian-like) $\mathcal{U}$ 's that already reside on the handlebody we can transport it onto any 1-handle of $\Sigma_{g}$. Cutting the lines going through $\mathcal{U}$ we can free $\mathcal{U}$ and next remove it. Thus $\mathcal{U L}^{-1}(T)$ consists of $\mathcal{L}^{-1}(T)$ and $g$-framed unknots (see Fig. 3). Then we 
obtain the following equality

$$
\left\langle\rho_{k}(h)\right\rangle_{0}=\left\langle\omega_{\mathcal{U}}\right\rangle^{-g}\left\langle\omega_{\mathcal{U} \mathcal{L}^{-1}(T)}\right\rangle,
$$

which expresses $(0,0)$-entry of $\rho_{k}(h)$, by means of a link invariant. The first term on RHS of (7) assures the right normalization of the $g \mathcal{U}$ 's. RHS of $(7)$ resembles Eq. 2, but there are some important differences. First of all, to repeat the Dehn twist construction of $\mathcal{M}$ in the surgery language, one is forced to put all the canonical surgery unknots $\mathcal{U}_{ \pm}$in the reversed order, i.e. according to Ref. 8, one should excavate tunnels in the handlebody $\mathcal{V}_{g}$ slightly deeper in each step. Fortunately, it appears that utilizing untwisted unknots $\mathcal{U}$ we can reverse the order of the canonical unknots $\mathcal{U}_{ \pm}$. The method is as follows. Temporarily adding auxiliary $\mathcal{U}$ 's (as it was explained earlier) we can cut out of the handlebody the two kinds of segments and corresponding graphs $G_{2}$ and $G_{4}$ (see Fig. 4). Turning the "ribbons" $180^{\circ}$ as shown in Fig. 4 we effectively change the order of lines. Gluing the lines back and throwing out auxiliary $\mathcal{U}$ 's we obtain $\mathcal{U L}(T)$, a link with the reversed order of all components (obviously, except $\mathcal{U}$ 's). Accordingly,

$$
\left\langle\omega_{\mathcal{U} \mathcal{L}^{-1}(T)}\right\rangle=\left\langle\omega_{\mathcal{U L}(T)}\right\rangle
$$

Gluing back the complementary handlebody $\mathcal{V}_{g}$ we obtain $\mathcal{M}$. Thus, by virtue of (6), (7) and (8),

$$
\phi_{k}(\mathcal{M})=\left\langle\omega_{\mathcal{U L}(T)}\right\rangle
$$

Since $\mathcal{U L}(T)$ provides us with a proper surgical description of $\mathcal{M}$, we should only account for the absence of the denominator present in Eq. 4. But

$$
\left\langle\omega_{\mathcal{U}_{ \pm}}\right\rangle=\kappa_{k}^{\mp(2 k+1)}
$$

and therefore the expressions (4) and (9) are equivalent modulo $\kappa_{k}^{(2 k+1) \sigma}$ (where $\sigma$ is the signature of the linking matrix $\ell k(\mathcal{L}))$, with $\mathcal{L}=\mathcal{U L}(T)$. 
In this way, we have shown that

$$
\tau_{k}(\mathcal{M})=\phi_{k}(\mathcal{M}) \bmod \Gamma_{k}
$$

for an arbitrary closed oriented 3-manifold $\mathcal{M}$.

\section{Acknowledgments}

The work has been partially supported by the KBN grants 2P30213906 and 2P30221706p01. When the work was in draft, the author received several papers independently addressing the same problem (see Ref. 9 and references therein).

\section{References}

1. E. Witten, Commun. Math. Phys. 121 (1989) 351.

2. N. Reshetikhin and V. G. Turaev, Invent. math. 103 (1991) 547.

3. F. Archer and R. M. Williams, Phys. Lett. B273 (1991) 438.

4. T. Kohno, Topology 31 (1992) 203.

5. T. Kohno, Intern. Journ. Mod. Phys. B6 (1992) 1795.

6. G. Moore and N. Seiberg, Commun. Math. Phys. 123 (1989) 177.

7. M. Li and M. Yu, Commun. Math. Phys. 127 (1990) 195.

8. D. Rolfsen, Knots and Links (Publish or Perish, Wilmington, 1976), Chapt. 9.

9. T. Kohno, Topological invariants for 3-manifolds using representations of mapping class groups II: Estimating tunnel number of knots, preprint 1992, submitted to Contemp. Math.;

J. Roberts, Math. Proc. Cam. Phil. Soc. 115 (1994) 53. 


\section{Figure captions}

Fig. $13 g-1$ canonical curves on a closed orientable 2-manifold $\Sigma_{g}$ of genus $g$.

Fig. 2 The annuli accommodating the circles corresponding to the Dehn twists and a dual graph representing a pant decomposition of $\Sigma_{g}$.

Fig. $3 \mathcal{U L}^{-1}(T)$ consisting of $\mathcal{L}^{-1}(T)$ and $g$ primary 0 -framed unknots shown in the lower part of Figure. There are also auxiliary 0-framed unknots in the upper part of Figure.

Fig. 4a $G_{2}$ cut out with 2 auxiliary 0 -framed unknots.

Fig. 4b $G_{4}$ cut out with 2 auxiliary and 2 primary 0-framed unknots. 


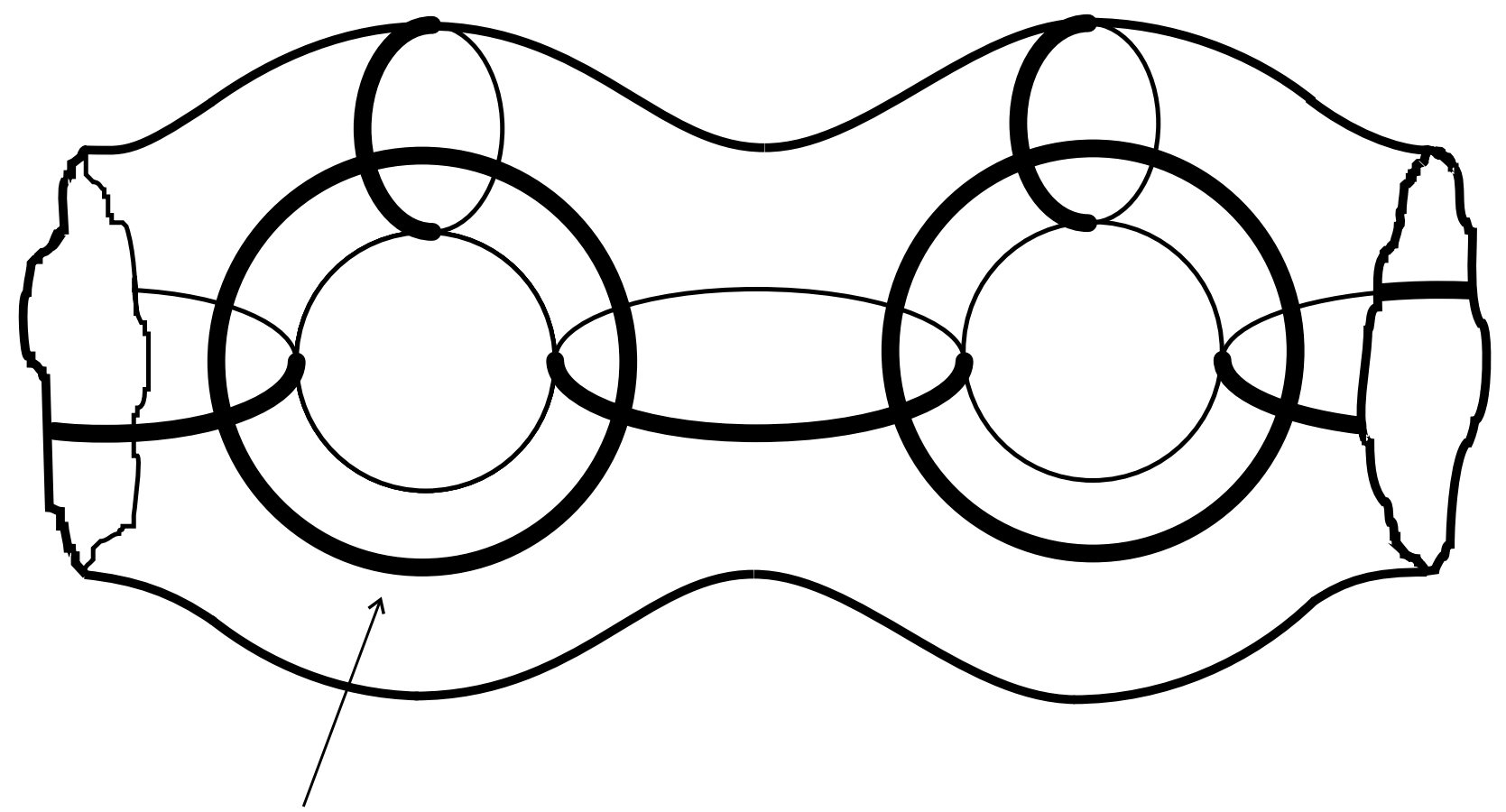

canonical curve

Fig. 1 


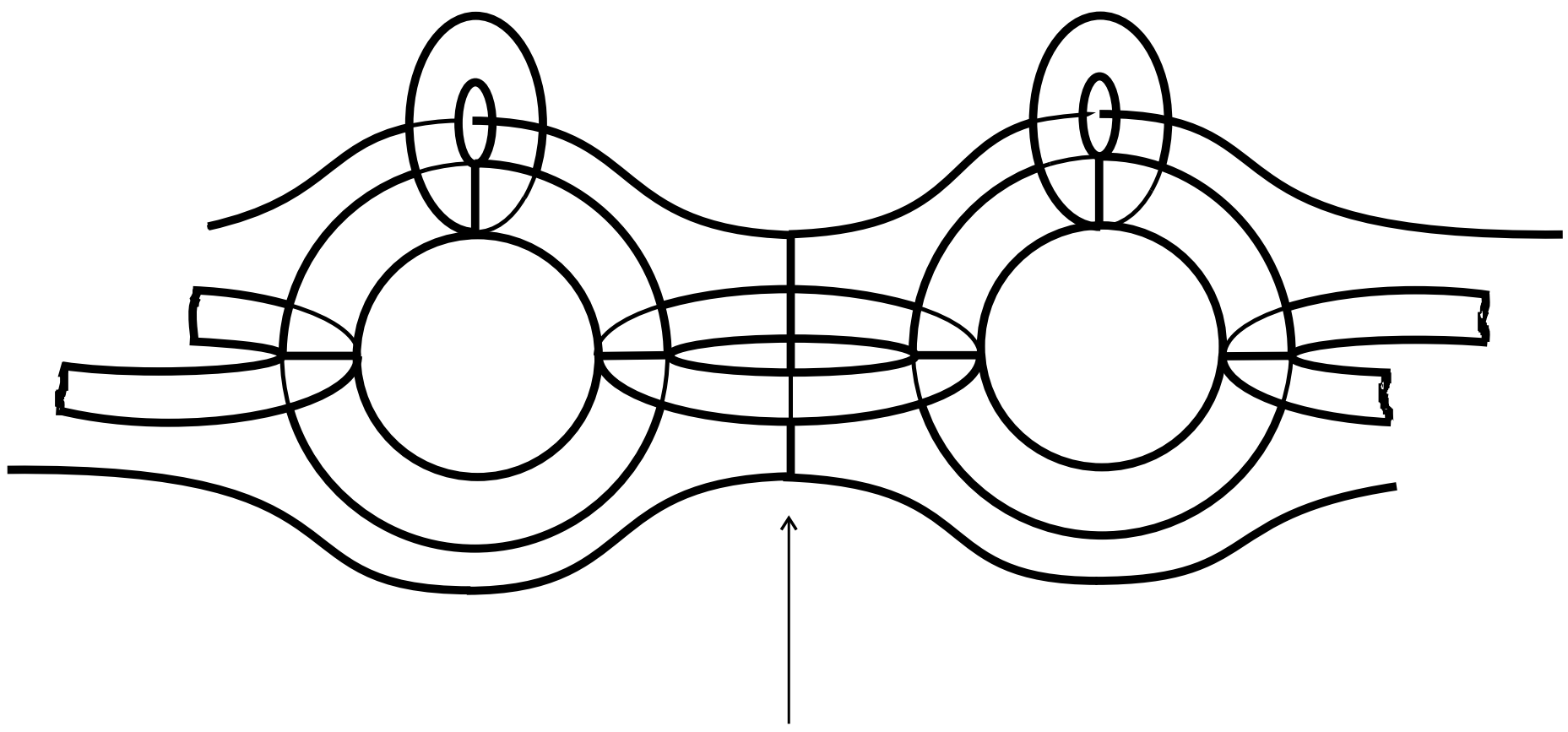

dual graph

Fig.2 


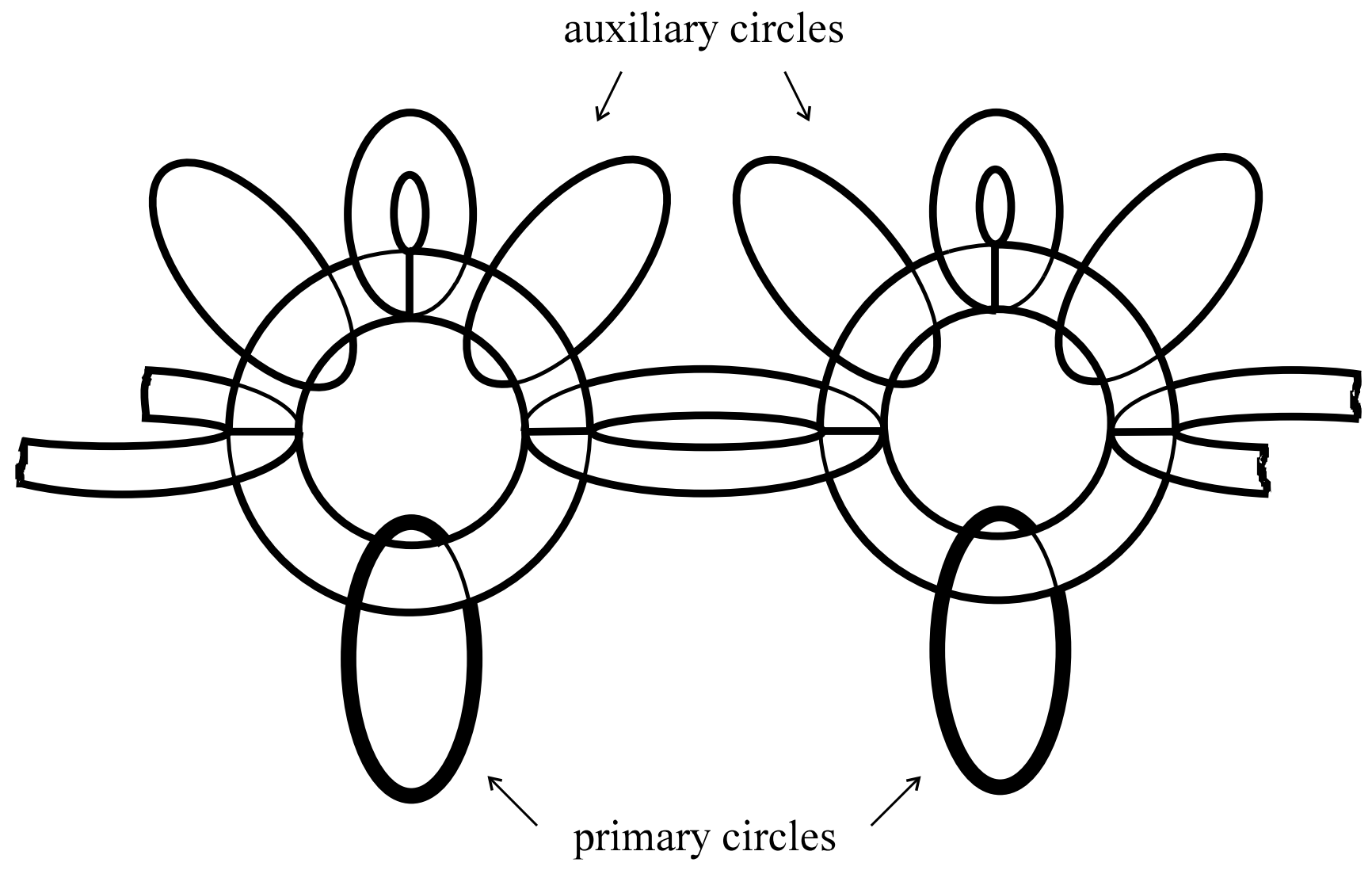

Fig. 3 


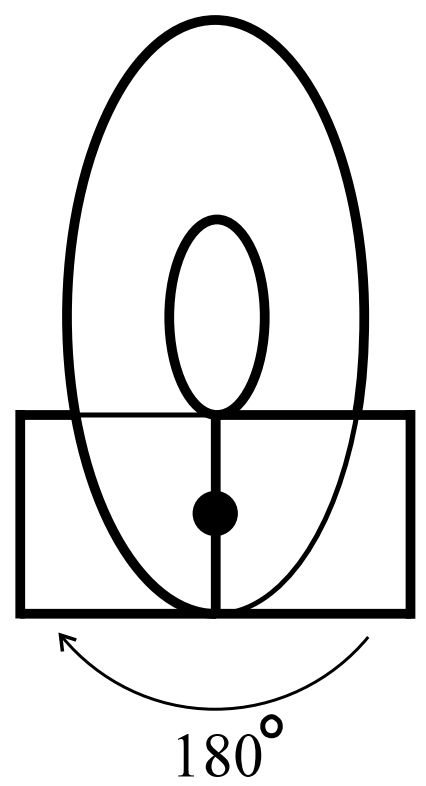

Fig. 4a 


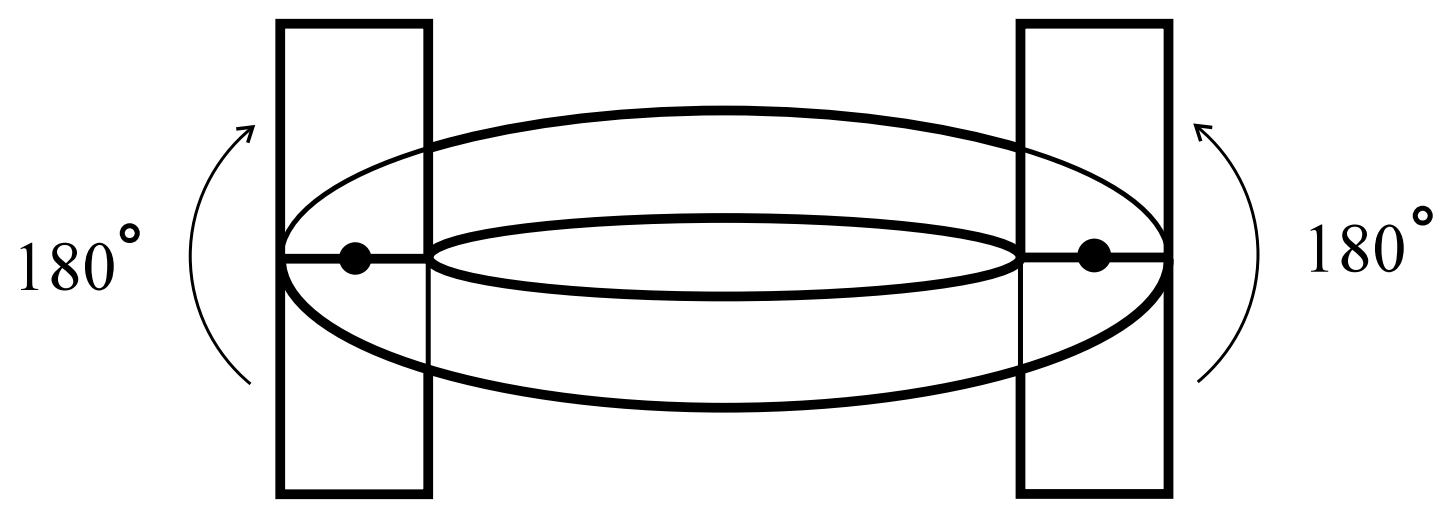

Fig. 4b 